\title{
SENSIBILIDADE DE ESPECTROS DE ONDAS OCEÂNICAS RECUPERADOS POR RADAR DE ABERTURA SINTÉTICA
}

\author{
Sensitivity of ocean-wave spectra retrieved by synthetic aperture radar
}

\author{
ANDRÉ LUIZ CORDEIRO DOS SANTOS ${ }^{1,2}$ \\ FELIPE MARQUES DOS SANTOS ${ }^{2}$ \\ LUIZ MARIANO CARVALHO2,3 \\ NELSON VIOLANTE-CARVALHO ${ }^{4}$ \\ ${ }^{1}$ Centro Federal de Educação Tecnológica do Rio de Janeiro, Departamento de Ensino Superior \\ Avenida Maracanã 229, Maracanã, CEP 20271-110, Rio de Janeiro - Brasil \\ andreluiz.cordeiro@gmail.com \\ 2 Universidade do Estado do Rio de Janeiro, Programa de Pós Graduação em Engenharia Mecânica. \\ Rua Fonseca Teles 121, São Cristóvão, CEP 20940-903, Rio de Janeiro - Brasil \\ felipems@gmail.com \\ ${ }^{3}$ Universidade do Estado do Rio de Janeiro, Instituto de Matemática \\ Rua São Francisco Xavier, 524, 4015E, CEP 20550-900 Rio de Janeiro, RJ, Brasil \\ luizmc@gmail.com \\ ${ }^{4}$ Universidade Federal do Rio de Janeiro, Programa de Engenharia Oceânica \\ Av. Athos da Silveira Ramos, 274. Cidade Universitária, Ilha do Fundão, CEP 21941-916, Rio de \\ Janeiro, RJ, Brasil \\ violante_carvalho@yahoo.co.uk
}

\section{Resumo:}

SAR (Synthetic Aperture Radar ou Radar de Abertura Sintética) é o único sensor transportado por satélites capaz de medir o espectro direcional de ondas. Sua elevada cobertura espacial e temporal permite caracterizar o estado de mar, especialmente a região de baixa frequência do espectro de energia, que vem sendo assimilada em modelos numéricos de previsão de ondas em diversos centros operacionais espalhados pelo globo. Contudo, a extração do espectro de ondas de uma imagem SAR é um procedimento complexo. Alguns modos de operação não permitem o emprego de imagens sequenciais para resolver a ambiguidade direcional de propagação das ondas, o que requer informações adicionais, geralmente obtidas de um modelo de ondas. A dependência destas informações adicionais é investigada aplicando-se a inversão clássica de Hasselmann a alguns estados de mar teóricos. Esta abordagem é baseada na transformação analítica do espectro direcional de ondas sobre o espectro de imagem SAR correspondente. A 
solução deste problema inverso é determinada por um algoritmo numérico que minimiza um funcional não linear. Apesar de amplamente utilizado por diversos centros operacionais de previsão, este método não foi extensivamente testado em cenários experimentais bem definidos. Os resultados mostram que a dependência investigada é bastante significativa, sobretudo no que diz respeito à direção de propagação das ondas, levantando questionamentos sobre a acurácia da técnica.

Palavras-chave: Espectro de Ondas; Sensibilidade; Radar de Abertura Sintética.

\begin{abstract}
:
Synthetic Aperture Radar (SAR) is the only sensor to date on-board satellites able to measure the directional wind wave spectrum. Its high spatial and temporal coverage allows the characterization of the sea state, especially at the low frequency region of the energy spectrum, which has been assimilated into numerical models at several operational centres world wide since the late 1990's. However, the extraction of the wave spectrum from a SAR image is a complex task. Not all imaging modes allow the use of sequential images to resolve the wave directional ambiguity, requiring some sort of additional, first guess information, in general from a wave model. The dependency of the wave retrieval on this first guess is investigated applying the classical Hasselmann inversion to some simple, theoretical, sea states. This approach is based on the analytical transformation relating the wind wave variance spectrum to its correspondent SAR image spectrum. The solution of this inverse problem is determined by a numerical algorithm, which minimizes a non-linear function. Although widely employed at operational forecasting centres, the method has not been extensively tested in experimental, well defined, situations. The results show that the first guess wave direction is fundamental to the reliability of the results obtained, raising questions about the accuracy of the technique.
\end{abstract}

Keywords: Ocean Wave Spectra; Sensitivity; Synthetic Aperture Radar.

\title{
1. INTRODUÇÃO
}

Desde o advento do radar de abertura sintética (SAR, da sigla em inglês synthetic aperture radar), transportado em satélites, uma quantidade considerável de dados do espectro direcional de ondas tornou-se disponível para cientistas. Atualmente, SAR é o único instrumento transportado em satélites capaz de estimar o espectro direcional e, portanto, de fornecer uma caracterização completa do estado do mar. Com elevada resolução espacial e temporal, os espectros de ondas obtidos de imagens SAR vêm sendo operacionalmente assimilados por modelos numéricos implementados em diversos centros de previsão (Aouf et al 2006, Heiberg et al 2006, Abdalla et al 2006). A possibilidade de medição do espectro direcional por uma extensa área, de até algumas dezenas de quilômetros quadrados, possibilita também uma série de investigações sobre as transformações espaciais sofridas pelas ondas (Violante-Carvalho et al 2012, Wang et al 2012, Collard et al 2005).

Existem diversos mecanismos que influenciam a estimativa do espectro direcional de ondas através de um radar de abertura sintética. A análise e a interpretação destes mecanismos dependem fortemente da compreensão das interações entre as ondas oceânicas e as ondas eletromagnéticas emitidas pelo radar. Diversos modelos interpretativos foram propostos (Kasilingam; Shemdin, 1990), porém, um modelo que explique definitivamente o complicado fenômeno que caracteriza a formação de imagens SAR não é conhecido no momento. A estimativa do espectro de ondas a partir de imagens SAR representa um problema desafiador, 
em que a maior dificuldade provém do comportamento aleatório da superfície oceânica ao longo do tempo (Phillips, 1977). Apesar disto, a análise espectral de imagens SAR demonstra que, sob condições específicas, é possível obter o espectro direcional de ondas de grandes comprimentos.

Hasselmann e Hasselmann (1991) propuseram uma relação funcional entre o espectro de imagem SAR e o espectro de ondas da correspondente superfície oceânica. Esta relação deve ser invertida para fornecer uma estimativa do espectro de ondas da correspondente imagem SAR. Entretanto, esse mecanismo de inversão não é único devido à ambiguidade direcional de $180^{\circ}$ inerente ao espectro congelado da imagem e a perda de informação do espectro de imagem para frequências acima do cut-off azimutal do SAR (Violante-Carvalho et al, 2012). Segundo os autores, os movimentos orbitais induzidos por ondas geram distorções não lineares na intensidade da imagem. Isto acarreta perda de informação na direção azimutal (paralela ao deslocamento do satélite) a partir de uma região de altas frequências, dando origem ao chamado cut-off azimutal. Por conta destas limitações apontadas, a inversão da relação de mapeamento espectral oceano-SAR, que é implementada, será viável apenas quando houver um espectro de ondas inicial (first-guess) disponível. Isto implica que a aplicação de uma imagem SAR para esta finalidade, em geral, emprega um modelo de ondas operacional para obter um espectro de ondas inicial. Portanto, a técnica de inversão de Hasselmann recupera um espectro de ondas associado à imagem SAR observado que é dependente da escolha do espectro de ondas inicial usado como dado de entrada do mecanismo de inversão. O principal objetivo deste artigo é estudar a influência do espectro de ondas inicial sobre o referido mecanismo.

A motivação para este trabalho vem dos artigos de Corsini e Manara (1995) e Litovchenko e Ivanov (1993). Corsini fez estimativas de espectros de ondas recuperados de imagens SAR simuladas, enquanto Litovchenko analisou parâmetros de ondas obtidos por satélites que orbitam em altitudes diferentes, ambos utilizando o procedimento de inversão de Hasselmann. Neste artigo, aplica-se o procedimento de inversão a um espectro de imagem SAR simulado, obtido da parametrização de um espectro de ondas unimodal, aqui denominado espectro de ondas de referência. O papel do espectro de ondas de referência é representar um estado de mar idealizado. Este espectro de ondas será usado para comparar resultados obtidos pelo algoritmo de inversão quando rotações do espectro de ondas de referência são utilizadas como dado de entrada (espectro de ondas inicial) do mecanismo de inversão. Deste modo, é possível determinar o grau de correlação entre o espectro de ondas de referência e o espectro de ondas recuperado em função da direção associada ao período de pico, aqui sendo chamada de direção principal do espectro. A correlação fornece um indicativo sobre a distorção que é imposta ao espectro de ondas recuperado, que é dependente do espectro de ondas inicial usado como dado de entrada do mecanismo de inversão. Um espectro de ondas recuperado é considerado muito

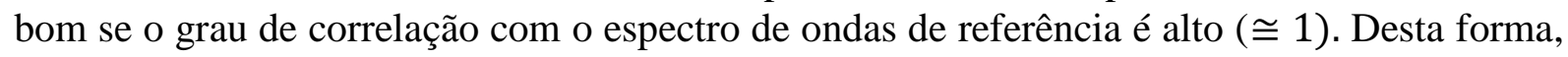
o espectro de ondas recuperado é bastante similar ao espectro de ondas de referência, que é, em teoria, a melhor resposta possível esperada do mecanismo de inversão. Paralelamente a isto, são avaliados os impactos sobre os parâmetros de ondas associados ao espectro de ondas recuperado, de modo a refinar ainda mais a análise.

É importante salientar que, para a execução desta proposta, foi necessário o desenvolvimento de uma complexa ferramenta computacional, aqui denominada de processador espectral. Este processador transforma um espectro de ondas oceânicas no correspondente espectro de imagem SAR e, portanto, constitui um ingrediente fundamental neste contexto: dispensa a necessidade de uma imagem SAR obtida de um satélite ou de um simulador de imagens. Acoplado ao processador espectral, é utilizado o algoritmo responsável pela inversão, doravante chamado simplesmente de algoritmo MPI (Max Plank Institute), desenvolvido na instituição que lhe dá o nome (Hassemann; Hasselmann, 1991). Há outras rotinas menores, porém importantes, envolvidas neste procedimento as quais serão destacadas oportunamente ao longo do texto. 
Não são conhecidos trabalhos publicados em periódicos cuja abordagem aqui proposta tenha sido utilizada para medir a distorção do espectro de ondas recuperado pelo mecanismo de inversão de Hasselmann. Nota-se que devido à complexidade do processo de formação de imagem SAR, simulações numéricas constituem uma importante ferramenta para uma melhor compreensão do processo de recuperação de espectro de ondas através de radares de abertura sintética.

Por fim, apresenta-se a estrutura do artigo: na seção 2, o SAR e seus mecanismos fundamentais são brevemente discutidos. Na seção 3, o algoritmo de inversão e o modelo teórico utilizado são descritos. Também são apresentados os conceitos de correlação espectral e de desvios (dos parâmetros de ondas), além do esquema numérico correspondente ao modelo teórico. $\mathrm{O}$ algoritmo utilizado para a realização das simulações é descrito na seção 4. Na seção 5, apresentam-se as conclusões.

A discussão apresentada na sequência tem como principal objetivo explicar a razão da simulação de ondas se propagando em três direções distintas em relação ao deslocamento do satélite: ondas se propagando na direção de range (ortogonal em relação ao deslocamento do satélite), na direção azimutal (paralela ao deslocamento do satélite) e numa direção intermediária às anteriores ( $45^{\circ}$ em relação ao deslocamento do satélite). Isto isolará a dependência das três modulações fundamentais introduzidas a seguir.

\section{SAR: MECANISMOS FUNDAMENTAIS}

Os conceitos apresentados ao longo desta seção podem ser encontrados em, por exemplo, Robinson (2004). No oceano são observadas ondas com uma grande variedade de comprimentos. De ondas centimétricas, geradas quando o vento começa a enrugar uma superfície espelhada, até ondas de algumas centenas de metros, típicas de ondulações. O pulso eletromagnético emitido pelo radar SAR, apresentando comprimento de onda de alguns centímetros, interage com as ondas presentes na superfície do oceano com comprimentos de mesma ordem, em um mecanismo denominado ressonância de Bragg. De forma geral, o sinal eletromagnético retroespalhado é proporcional à amplitude destas ondas centimétricas, denominadas ondas de Bragg, sempre presentes na superfície do oceano caso haja ventos de intensidade mínima de $3 \mathrm{~m} / \mathrm{s}$ aproximadamente.

As ondas de Bragg são as ondas efetivamente medidas pelo radar, porém apresentam menor importância do ponto de vista prático quando comparadas às ondas de maior comprimento e com maior energia. Estas ondas de maior comprimento se tornam detectáveis pelo radar uma vez que modulam as ondas de Bragg em um mecanismo chamado de duas escalas (two-scale model). Basicamente são três os mecanismos de modulação das ondas de Bragg que tornam as ondas de maior comprimento observáveis pelo SAR:

(m1) modulação de inclinação - altera o ângulo de incidência da energia emitida pelo satélite em função da esbeltez das ondas de maior comprimento.

(m2) modulação hidrodinâmica - advém das zonas de convergência e divergência de ondas de Bragg decorrentes dos movimentos orbitais induzidos por ondas de maior comprimento.

(m3) velocity bunching - efeito oriundo do mecanismo de abertura sintética empregada pela técnica SAR. Para simular uma antena de maior abertura, e consequentemente aumentar a resolução espacial, o desvio Doppler do sinal de um mesmo alvo mapeado em momentos distintos do deslocamento do satélite é empregado para determinar sua posição no plano da imagem. Como a superfície do oceano é um alvo não estático devido ao movimento orbital induzido pela presença das ondas de maior comprimento, este movimento induz uma alteração 
na frequência do sinal retroespalhado. Dependendo da esbeltez das ondas, esta alteração se torna altamente não linear impossibilitando a correção da posição do alvo no plano da imagem. A modulação de inclinação e a modulação hidrodinâmica são responsáveis pela detecção das ondas se propagando em uma direção ortogonal ao deslocamento do satélite (direção de range), enquanto que o velocity bunching é responsável pela detecção de ondas se propagando paralelamente à direção de deslocamento do satélite (direção azimutal).

\section{METODOLOGIA}

\subsection{Algoritmo de Inversão}

O estado do mar pode ser estatisticamente caracterizado pelo espectro bidimensional de ondas, o qual descreve a distribuição da energia da onda com respeito à direção de propagação e sua frequência. A primeira etapa na definição de um procedimento numérico para recuperação de espectro de ondas a partir de imagens SAR é determinar a relação entre a variância da intensidade da imagem SAR e a variância da elevação das ondas do mar. Estas variâncias estão relacionadas através da transformação H (Hasselmann; Hasselmann, 1991; Krogstad; Shyberg, 1992), de tal forma que

$$
\mathrm{H}(P(\boldsymbol{k}))=S_{\text {sar }}(\boldsymbol{k})
$$

Em (1), $P$ e $S_{\text {sar }}$ denotam, respectivamente, o espectro de ondas e o espectro de imagem SAR, sendo $\boldsymbol{k}$ o vetor número de onda. A transformação $\mathrm{H}$ é denominada transformada de Hasselmann, uma aplicação fortemente não linear que carrega todas as informações sobre a modulação SAR através das funções de transferência de modulação. A hipótese fundamental feita por Hasselmann, a qual permitiu estabelecer a relação (1), foi supor que a modulação SAR (modulação de inclinação + modulação hidrodinâmica + velocity bunching) está linearmente relacionada com a elevação do oceano através de filtros, representados por suas respectivas funções de transferência (Krogstad; Shyberg, 1992).

Basicamente, o procedimento de inversão consiste na obtenção do espectro direcional de ondas a partir uma de imagem SAR. Entretanto, esse mecanismo de inversão não é único devido à ambiguidade direcional de $180^{\circ}$ inerente ao espectro congelado da imagem e à perda de informação do espectro de imagem para frequências acima do cut-off azimutal do SAR. Estas dificuldades são contornadas através de um espectro de ondas inicial (first-guess), utilizado para decidir a questão direcional, bem como de fonte de informação para estender às altas frequências, ou seja, frequências acima do cut-off azimutal.

Em termos teóricos, este procedimento é realizado pela minimização do seguinte funcional (Hasselmann; Hasselmann, 1991):

$$
\boldsymbol{J}(P)=\int\left[S_{\text {sar }}(\boldsymbol{k})-S_{o b s}(\boldsymbol{k})\right]^{2} d \boldsymbol{k}+\mu \int \frac{\left[P(\boldsymbol{k})-P^{0}(\boldsymbol{k})\right]^{2}}{[B+P(\boldsymbol{k})]^{2}} d \boldsymbol{k}
$$

Em (2), $P^{0}$ e $S_{\text {obs }}$ representam o espectro de ondas inicial e o espectro de imagem SAR observado respectivamente. A constante $\mu$, que possui uma limitação apresentada adiante, é escolhida de acordo com a confiabilidade do espectro de imagem SAR observado relativamente ao espectro de ondas inicial. $B$ é uma constante positiva com valor pequeno, introduzida para prevenir que o denominador da segunda integral não se anule. 
Seja $P^{r}$ o espectro de ondas que satisfaz a desigualdade

$$
J\left(P^{r}\right) \leq J(P)
$$

qualquer que seja o espectro de ondas $P$. De acordo com (2), o espectro de ondas $P^{r}$ é recuperado da informação fornecida pelo espectro de imagem SAR observado $S_{o b s}$ e pelo espectro inicial de ondas $P^{0}$. Por esta razão, $P^{r}$ é denominado espectro de ondas recuperado. Segundo Komen (1994), a condição de mínimo local,

$$
\frac{\partial J}{\partial P}=0
$$

satisfeita por $P^{r}$, é obtida iterativamente através do algoritmo MPI (Max Plank Institute). Este algoritmo baseia-se em aplicações alternadas da transformação não linear H no sentido oceanoSAR e, da forma simplificada (linear) de H na inversão, isto é, no sentido SAR-oceano. A convergência do esquema iterativo a um resultado satisfatório depende da semelhança entre o espectro de ondas inicial e o espectro de ondas de referência. Desta forma, o espectro de ondas inicial, utilizado para inicializar o processo iterativo, exerce um papel fundamental neste processo. Entretanto, o esquema de iterações do algoritmo MPI converge mesmo nos casos em que o espectro de ondas inicial é pouco confiável, desde que $\mu$ não seja tão pequeno, isto é, $\mu \geq$ $\mu_{0}$ em que

$$
\mu_{0}=0,1 \max S_{o b s}^{2}
$$

e a constante $B$ seja tomada como sendo 0,01 max $\mathrm{P}^{0}$ (Hasselmann; Hasselmann, 1991). Nestas condições, nem todo resultado obtido pelo mecanismo retratará com fidelidade o estado de mar considerado. Isto motiva tentar compreender em que medida um espectro de ondas inicial influencia o resultado obtido pelo algoritmo de inversão.

\subsection{Modelo Teórico}

Num cenário operacional, o SAR faz a aquisição de imagens do oceano e, após adequado processamento de dados, o espectro de imagem SAR é obtido. Este espectro de imagem, junto com a informação prévia fornecida pelo espectro de ondas inicial, constituem os dados de entrada requeridos para a execução do algoritmo MPI. Este algoritmo fornecerá como resposta o espectro direcional de ondas associado à cena observada pelo satélite (isto é, o espectro de ondas recuperado). Devido ao processador espectral desenvolvido pelo grupo, um dos avanços obtidos neste trabalho é a possibilidade de produzir artificialmente espectros de imagens SAR a partir de parâmetros de onda escolhidos previamente. Isto abre caminhos para a realização de experimentos com grande controle sobre todos os parâmetros envolvidos neste complexo mecanismo de recuperação de espectros direcionais de onda.

Neste estudo, a execução teórica do procedimento considera os três dados de entrada enumerados a seguir:

(i1) o espectro direcional de ondas de referência: $P$

(i2) o espectro direcional de ondas inicial: $P^{0}$

(i3) o espectro de imagem observado: $\widetilde{S_{\text {obs }}}$

$\mathrm{O}$ espectro de ondas de referência $P$, gerado sinteticamente, caracteriza um estado de mar idealizado. Seria este o estado de mar, em teoria, observado pelo satélite. É a partir deste espectro de ondas de referência que se obtém $\widetilde{S_{o b s}}$, o espectro de imagem SAR observado. O espectro de ondas inicial $P^{0}$ também será obtido do espectro de ondas de referência $P$, conforme descrição feita adiante. 
Inicialmente, define-se o dado de entrada (i1). Para isto, combina-se um espectro de energia do tipo JONSWAP (Hasselmann, 1973) com uma distribuição direcional do tipo $\cos ^{2 s}$ (LonguetHiggins, 1957). Computacionalmente, isto é feito utilizando-se o pacote de rotinas WAFO (sigla do inglês: Wave Analysis for Fatigue and Oceanography. WAFO-group, 2000), desenvolvido para análise e simulação de dados de onda. Essas rotinas requerem três parâmetros de onda como entrada: altura significativa $\left(H_{m_{0}}\right)$, período de pico $\left(T_{p}\right)$, direção principal $\left(\theta_{w}\right)$, além da escolha do tipo de distribuição direcional.

Em seguida, o dado de entrada (i2) pode ser definido através do espectro de ondas de referência $P$, obtido em (i1), bastando para isto efetuar adequadas perturbações dos parâmetros de ondas exigidos pelo pacote WAFO e usando-se a mesma distribuição direcional de $P$.

Finalmente, define-se o dado de entrada (i3). Em tese, esta informação é obtida através de uma aplicação direta da transformação de Hasselmann sobre (i1), o espectro de ondas de referência, isto é, $\mathrm{H}(P(\boldsymbol{k}))=S_{\text {obs }}$. A obtenção do espectro de imagem SAR observado utiliza o processador espectral, citado anteriormente. Este processador é a versão computacional da transformada de Hasselmann. Esta complexa ferramenta foi implementada em Matlab e desempenha papel destacado neste estudo, pois é responsável por gerar um espectro de imagem SAR sintético a partir de um estado de mar plenamente conhecido, dispensando o uso de uma imagem SAR para realização de experimentos.

É fundamental observar que, de acordo com o procedimento descrito, os dados de entrada (i2) e (i3) são obtidos de forma que as condições de similaridade entre os espectros referência e inicial possam ser controladas, assim como a confiabilidade do espectro de imagem SAR relativamente ao espectro de ondas inicial. De acordo com Robinson (2004), num processo operacional de aquisição de dados pelo SAR, o sinal que retorna ao radar é contaminado por ruído (speckle). O algoritmo MPI foi, por isso, concebido para receber um espectro de imagem SAR ruidoso, de modo que deve-se levar este fato em consideração. Desta forma, o espectro de imagem SAR observado, produzido sinteticamente, será dado por

$$
\widetilde{S_{o b s}}(\boldsymbol{k})=\mathrm{H}(P(\boldsymbol{k}))+N(\boldsymbol{k})
$$

em que $N$ simula o efeito de speckle. Maiores detalhes sobre a introdução deste ruído gerado randomicamente serão apresentados mais adiante.

\subsection{Conceituação}

É importante definir quantidades que permitam medir a influência do espectro de ondas inicial - usado como dado de entrada pelo algoritmo MPI - sobre o espectro direcional recuperado. Neste sentido, considera-se o espectro de ondas de referência $P$ associado a um estado de mar idealizado, o espectro de imagem SAR observado $\widetilde{S_{\text {obs }}}$ correspondente à cena descrita por $P \mathrm{e}$ um espectro de ondas inicial $P^{0}$, todos obtidos conforme (i1), (i2) e (i3). A partir de agora, supõe-se, sem menção explícita, que $P^{r}$ é o espectro de ondas recuperado pelo algoritmo MPI quando utilizados o espectro de imagem $\widetilde{S_{o b s}}$ e o espectro de ondas inicial $P^{0}$ como dados de entrada.

\subsubsection{Correlação Espectral}

Um conceito importante e amplamente utilizado na comparação de imagens é o conceito de correlação. Intuitivamente, duas imagens são correlacionadas se elas são semelhantes entre si, 
e não são correlacionadas se elas são bastante diferentes. Por exemplo, duas cópias idênticas da mesma imagem são totalmente correlacionadas, mas adicionando-se gradualmente um ruído aleatório a uma delas, a correlação irá reduzir, até que, finalmente, tornem-se não correlacionadas. Assim, o grau de correlação fornece uma indicação para a semelhança entre as imagens.

Esta ideia pode ser adaptada ao presente contexto, usando-a para medir o grau de similaridade (correlação) entre o espectro de ondas referência e o espectro de ondas recuperado pelo SAR. Baseados em Cartwright (1990), define-se o grau de similaridade - devido ao espectro de ondas inicial $P^{0}$ - entre o espectro de ondas de referência $P$ e o espectro de ondas recuperado $P^{r}$, como sendo

$$
g\left(P^{0}\right)=\frac{P}{\|P\|} \circ \frac{P^{r}}{\left\|P^{r}\right\|}
$$

Em (7), o símbolo ${ }^{\circ}$ denota o produto interno de Frobenius entre matrizes, enquanto que o símbolo \|. .| denota a norma matricial induzida por este produto interno (Stewart, 2011). O grau de similaridade (coeficiente de correlação) entre o espectro de ondas de referência e o espectro de ondas recuperado será máximo quando for igual a 1 (correlação total) e mínimo se for igual 0 (não há correlação).

\subsubsection{Parâmetros Espectrais}

A fim de atingir uma compreensão maior acerca do espectro de ondas recuperado, é importante distinguir o impacto do espectro de ondas inicial sobre cada um de seus parâmetros de onda. Assim, considerando $P$, o espectro de ondas de referência, com parâmetros espectrais

$$
\left\{H_{m_{0}}, T_{p}, \theta_{w}, \theta_{m}\right\}
$$

altura significativa, período de pico, direção principal e direção média respectivamente, e $P^{r}$, o espectro de ondas recuperado, com parâmetros espectrais (ordenados como acima)

$$
\left\{H_{m_{0}}^{r}, T_{p}^{r}, \theta_{w}^{r}, \theta_{m}^{r}\right\}
$$

definem-se os desvios associados aos parâmetros espectrais do seguinte modo:

(I) Desvio da altura significativa:

$$
D_{H}\left(P^{0}\right)=\frac{\left|H_{m_{0}}-H_{m_{0}}^{r}\right|}{H_{m_{0}}}
$$

(II) Desvio do período de pico:

$$
D_{T}\left(P^{0}\right)=\frac{\left|T_{p}-T_{p}^{r}\right|}{T_{p}}
$$

(III) Desvio da direção principal:

$$
D_{\theta_{w}}\left(P^{0}\right)=\min \{\lambda, 2-\lambda\}
$$


em que $\lambda=\frac{\left|\theta_{w}-\theta_{w}^{r}\right|}{180}$

(IV) Desvio da direção média:

$$
D_{\theta_{m}}\left(P^{0}\right)=\min \{\beta, 2-\beta\}
$$

em que $\beta=\frac{\left|\theta_{m}-\theta_{m}^{r}\right|}{180}$.

As definições (formais) dos parâmetros espectrais podem ser encontradas em Kahma (2005). A densidade de energia espectral, que é proporcional ao quadrado da altura significativa, também será considerada a fim de complementar as análises.

\subsubsection{Esquema Numérico}

A meta fundamental é estudar o espectro direcional de ondas recuperado de um espectro de imagem SAR (observado) quando utilizados espectros de ondas iniciais obtidos de rotações do espectro de ondas de referência, como dados de entrada do algoritmo MPI (de fato, uma mudança da direção principal do espectro de ondas de referência corresponde a uma rotação do mesmo). Neste sentido, considere o espectro de ondas de referência $P$ caracterizado pelos parâmetros em $(9)$, e seja $\widetilde{S_{\text {obs }}}(\boldsymbol{k})$, o espectro de imagem SAR (observado) associado à cena teórica descrita por $P$.

A sequência

$$
\left\{P_{j}^{0}:-12 \leq j \leq 12\right\}
$$

de espectros direcionais, cujos parâmetros: altura significativa, período de pico e direção principal indicados, respectivamente, por

$$
\left\{H_{m_{0}}^{0, j}, T_{p}^{0, j}, \theta_{w}^{0, j}\right\}
$$

são numericamente definidos pelas equações,

$$
\left\{\begin{array}{l}
H_{m_{0}}^{0, j}=H_{m_{0}} \\
T_{p}^{0, j}=T_{p} \\
\theta_{w}^{0, j}=\theta_{w}+15 j
\end{array}\right.
$$

é formada apenas de rotações do espectro de ondas de referência. É importante destacar que para valores de $j$ para os quais $\theta_{w}^{0, j}$ é negativo, deve-se considerar a menor determinação positiva deste ângulo, isto é, $\theta_{w}^{0, j}+360^{\circ}$. Em particular, $P_{0}^{0}$ é o próprio espectro de ondas de referência $P$.

Assim, após todas as rotações $P_{j}^{0}$ do espectro de ondas de referência $P$ serem utilizadas como dados de entrada do algoritmo de inversão, o mecanismo terá produzido uma sequência de espectros direcionais recuperados $\left\{P_{j}^{r}\right\}$ que, juntamente com seus parâmetros, denotados por, 


$$
\left\{H_{m_{0}}^{r, j}, T_{p}^{r, j}, \theta_{w}^{r, j}, \theta_{m}^{r, j}\right\}
$$

serão avaliados através dos indicadores (correlação e desvios) estabelecidos por (11), (12), (13) e (14).

\section{SIMULAÇÕES}

A partir de um espectro de imagem SAR, define-se que um experimento consiste na obtenção do espectro de ondas recuperado (e seus parâmetros) através do processo de inversão, uma vez que uma rotação do espectro de ondas de referência tenha sido utilizada como dado de entrada para iniciar o processo. Uma simulação é um conjunto de 25 experimentos, sendo cada um deles determinado em função da rotação imposta ao espectro de ondas de referência, conforme descrito em (17). Neste trabalho foram realizadas 3 simulações, perfazendo um total de 75 experimentos. Utilizou-se uma máquina com processador Intel Core i5-3210M 2.50GHz, 4GB de memória RAM com o sistema operacional Ubuntu 12.04.5 LTS (Precise Pangolin) 64 bits na execução das rotinas. O processador espectral está implementado em Matlab, enquanto que o algoritmo MPI está implementado em Fortran.

Os parâmetros de entrada utilizados no WAFO são: $H_{m_{0}}=4,8$ metros (altura significativa), $T_{p}=13$ segundos (período de pico), $s=15$ (coeficiente no espalhamento direcional cos $2 \mathrm{~s}$ ). Para simular o efeito speckle (ruído), foi criada uma matriz com entradas aleatórias e uniformemente distribuídas variando no intervalo $[0,10]$. Esta matriz é adicionada à matriz correspondente ao espectro de imagem SAR. Os parâmetros de satélite utilizados correspondem aos do ERS-1 (European Remote Sensing), que esteve em atividade até 2011.

Uma descrição detalhada do algoritmo utilizado nas simulações realizadas é apresentada a seguir:

1. Definição dos parâmetros para geração de um espectro direcional de ondas: altura significativa, $H_{m_{0}}$, período de pico, $T_{p}$, e direção principal, $\theta_{w}$.

2. Geração de um espectro direcional de ondas utilizando rotinas do WAFO adaptadas (espectro de ondas de referência).

3. Execução do processador espectral.

3.1. Geração de um espectro da imagem SAR associado ao espectro direcional de ondas gerado no item 2, utilizando a transformação não linear de Hasselmann oceano-SAR.

3.2. Adição de ruído ao espectro da imagem SAR.

3.3. Conversão do espectro da imagem para o padrão UWA da ESA.

4. Roda 25 experimentos baseados no arquivo gerado no item 3.3.

4.1. Para $\theta_{w}=0^{\circ}$ até $345^{\circ}$ com incrementos de $15^{\circ}$. Aqui há um loop entre os passos (4.1) e (4.2) onde o valor de $\theta_{w}$ é alterado, a partir de $0^{\circ}$ até chegar em $345^{\circ}$.

4.1.1. Utiliza os mesmos valores dos parâmetros $H_{m_{0}}$ e $T_{p}$ utilizados no item 1 .

4.1.2. Geração de um espectro direcional de ondas utilizando rotinas do WAFO adaptadas (espectro de ondas inicial).

4.1.3. Roda o MPI.

4.1.4. Armazena os resultados de forma conveniente (espectro de ondas recuperado + parâmetros de onda)

4.2. Fim para.

Bol. Ciênc. Geod., sec. Artigos, Curitiba, v. 21, no 2, p.251-273, abr-jun, 2015. 


\section{RESULTADOS}

Os gráficos ao longo desta seção devem ser entendidos a partir das seguintes instruções: (I) Cada unidade no eixo horizontal corresponde à direção principal do espectro de ondas inicial utilizado como dado de entrada do algoritmo de inversão (MPI); (II) A direção principal do espectro de ondas de referência é representada em $0^{\circ}$. Os espectros de ondas de referência considerados possuem as direções: range (ortogonal em relação ao deslocamento do satélite), azimutal (paralela ao deslocamento do satélite) e intermediária às anteriores ( $45^{\circ} \mathrm{em}$ relação ao deslocamento do satélite). (III) A direção do espectro de imagem SAR está representada no eixo horizontal por $0^{\circ}$ (que coincide com a direção principal do espectro de ondas de referência) e $\pm 180^{\circ}$ (ambiguidade); (IV) A variação angular da direção principal de um espectro de ondas inicial em relação à direção principal do espectro de ondas de referência será denotada por $\Delta^{0}$. A seguir, alguns exemplos ilustrarão como determinar a direção principal de um espectro de ondas inicial. Se a direção principal do espectro de ondas de referência for, por exemplo, $15^{\circ} \mathrm{e}$ variação angular $\Delta^{0}=75^{\circ}$, então a direção principal do espectro de ondas inicial é $90^{\circ}=$ $15^{\circ}+75^{\circ}$. Se a variação for $\Delta^{0}=-120^{\circ}$, então a direção principal do espectro de ondas inicial é $15^{\circ}-120^{\circ}=-105^{\circ}$. A menor determinação positiva deste arco é $360^{\circ}-105^{\circ}=$ $255^{\circ}$;

A fim de sistematizar a análise realizada, esta tarefa será dividida em partes.

\subsection{Direção Média}

Na Figura 1, cada curva corresponde ao desvio da direção média do espectro de ondas recuperado relativamente ao espectro de ondas de referência - definido em (14) - em função da direção principal do espectro de ondas inicial. Nota-se que todas as curvas estão sobrepostas e possuem um aspecto comum: a forma de sino invertido. Isto significa que a direção média é um parâmetro do espectro de ondas recuperado que é bastante sensível à variação da direção principal do espectro de ondas inicial, e este comportamento é observado em todas as direções dos espectros de ondas de referência considerados nas simulações. De fato, o desvio da direção média aumenta à medida que a variação angular $\Delta^{0}$ também aumenta. Por exemplo, para variações angulares $\left|\Delta^{0}\right| \leq 45^{\circ}$, o desvio da direção média é menor que $20 \%$. Em particular, o desvio da direção média é máximo (igual a 100\%) quando a direção principal do espectro de ondas inicial é oposta à direção principal do espectro de ondas de referência $\left(\left|\Delta^{0}\right|=180^{\circ}\right)$. Conclui-se, portanto, que a direção média do espectro de ondas recuperado acompanha as rotações imprimidas ao espectro de ondas de referência. 


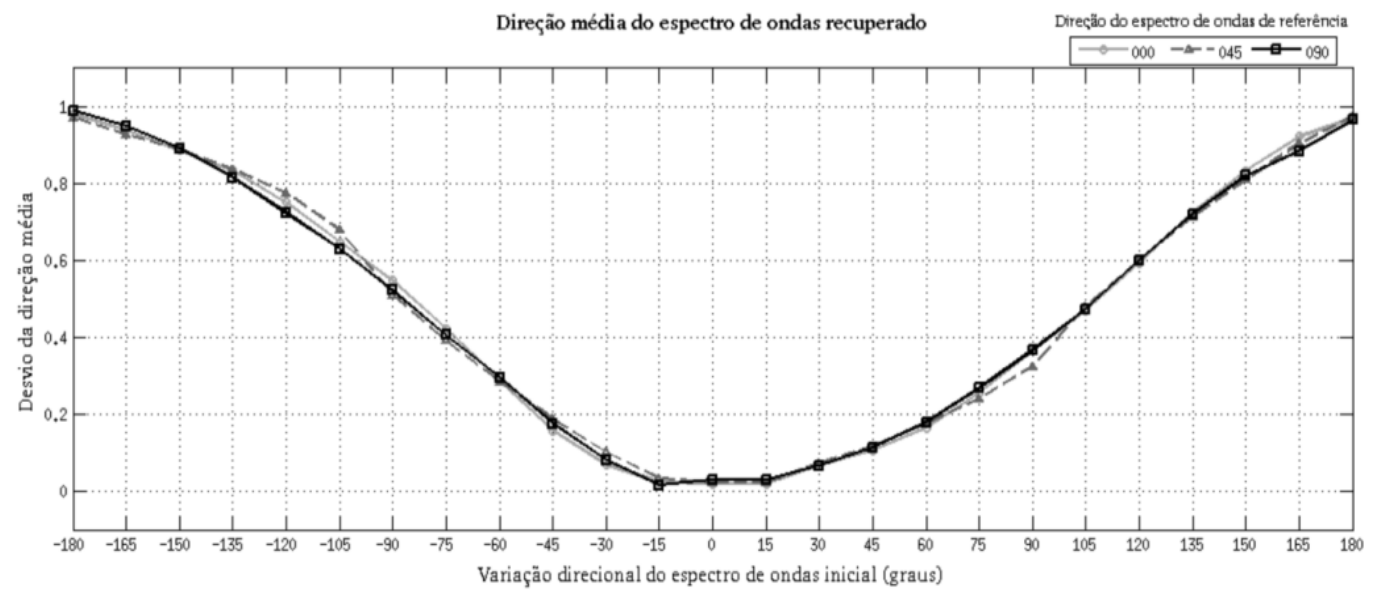

Figura 1: Representação da variação do desvio da direção média (adimensional) do espectro de ondas recuperado em função da direção principal do espectro de ondas inicial e da direção principal do espectro de referência relativamente a direção do voo do satélite.

\subsection{Direção Principal}

Na Figura 2, cada curva corresponde ao desvio da direção principal do espectro de ondas recuperado relativamente ao espectro de ondas de referência - definido em (13) - em função da direção principal do espectro de ondas inicial. Todas as curvas possuem um aspecto comum: a forma aproximada da função degrau. Isto caracteriza que a direção principal do espectro de ondas recuperado é pouco sensível às variações angulares da direção principal do espectro de ondas inicial. De fato, vê-se que se $\left|\Delta^{0}\right| \leq 60^{\circ}$, o desvio da direção principal do espectro de ondas recuperado é menor que $10 \%$. Por outro lado, para variações angulares tais que $\left|\Delta^{0}\right| \geq 75^{\circ}$, de um modo geral, o mecanismo de inversão fornece espectros recuperados com direções principais considerável ou absolutamente discrepantes da direção principal do espectro de ondas de referência. Isto é caracterizado pelo salto súbito evidenciado pelas curvas na faixa angular de $\left|\Delta^{0}\right| \cong 75^{\circ}$, pois verifica-se que a direção do espectro de ondas recuperado possui um desvio em torno $80 \%$ ou mais.

Esta discussão sugere que as repostas do algoritmo MPI, em relação à direção principal de propagação das ondas, são pouco sensíveis à direção do espectro de ondas inicial sendo determinadas principalmente pela direção do espectro de imagem SAR. De fato, considerandose que a direção do espectro de imagem observado está representada nos gráficos por $\Delta^{0}=0^{\circ}$ e $\Delta^{0}= \pm 180^{\circ}$, verifica-se que as curvas são estáveis nestas vizinhanças. Como contraponto, é bom lembrar que a direção média do espectro de ondas recuperado acompanha a variação da direção do espectro de ondas inicial, isto é, não há, como neste caso, estabilidade em relação às variações da direção principal do espectro de ondas inicial. 


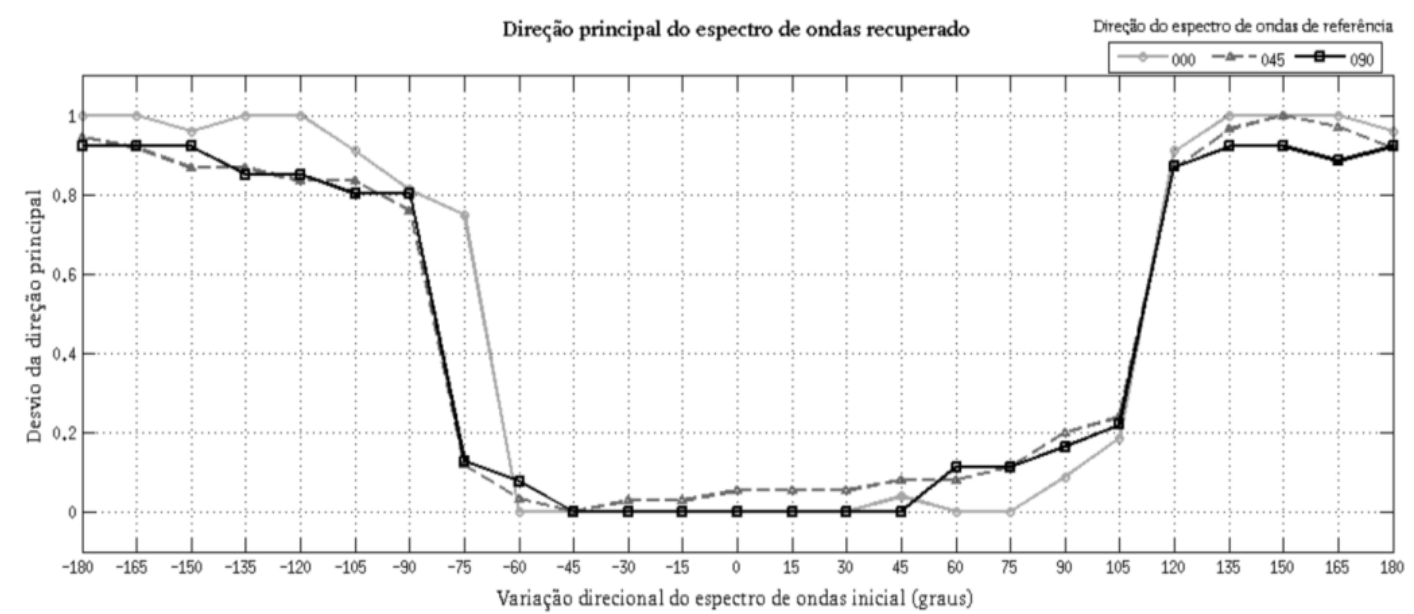

Figura 2: Representação da variação do desvio da direção principal (adimensional) do espectro de ondas recuperado em função da direção principal do espectro de ondas inicial e da direção principal do espectro de referência relativamente à direção do voo do satélite.

\subsection{Altura Significativa}

Na Figura 3-(a), cada curva corresponde ao desvio da altura significativa do espectro de ondas recuperado relativamente ao espectro de ondas de referência - definido por (11) - em função da direção principal do espectro de ondas inicial. Diferentemente do que acontece com os casos analisados anteriormente, não há, num primeiro momento, um evidente padrão comum a todas as curvas.

Analisando as curvas, observa-se que o desvio médio da altura significativa é influenciado pela direção principal do espectro de ondas de referência. Considerando a curva referente à direção azimutal $\left(0^{\circ}\right)$, o desvio médio da altura significativa é aproximadamente de $17.4 \%$. No caso da curva referente à direção intermediária $\left(45^{\circ}\right)$, verifica-se um desvio médio da altura significativa de aproximadamente $12 \%$. E, por fim, no caso da curva referente à direção de range $\left(90^{\circ}\right)$, o desvio médio da altura significativa é de aproximadamente $5 \%$. Isto revela que o desvio da altura significativa torna-se menor à medida que a direção principal das ondas se aproxima da direção de range (ortogonal ao deslocamento do satélite).

Nas Figuras 3-(b), 3-(c) e 3-(d), observa-se como varia a densidade média de energia dos espectros recuperados de acordo com a direção principal do espectro de ondas inicial, para ondas se propagando, respectivamente, nas direções azimutal, intermediária $\left(45^{\circ}\right)$ e de range. É possível notar um comportamento comum nesses gráficos: de modo geral, os maiores valores da densidade de energia são percebidos à medida que a direção principal do espectro de ondas inicial tende a alinhar-se à direção do espectro de imagem SAR. Isto ocorre quando a variação angular $\Delta^{0}$ estiver na vizinhança de $0^{\circ}$ ou $\pm 180^{\circ}$. Nota-se ainda que, nestas vizinhanças, os valores da densidade de energia aumentam quanto menor for o desvio angular entre a direção de propagação das ondas e a direção azimutal. Devido à relação entre a densidade média de energia das ondas e a altura significativa das ondas, esta análise mostra que a altura significativa é sensível à variação da direção principal do espectro de ondas inicial e tem um comportamento muito bem determinado nas condições consideradas. 

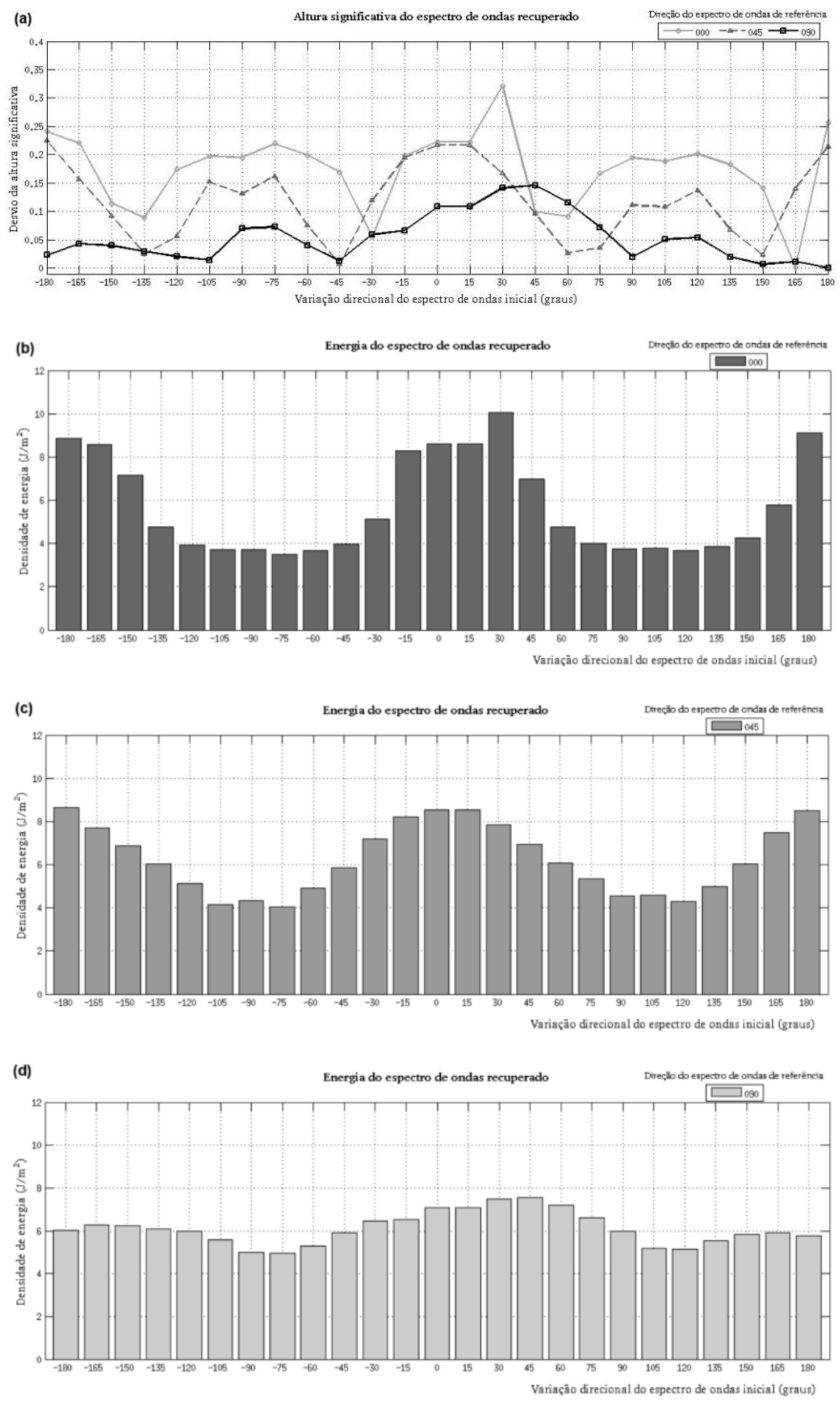

Figura 3: (a) Representação da variação do desvio da altura significativa (adimensional) do espectro de ondas recuperado em função da direção principal do espectro de ondas inicial e da 
direção principal do espectro de referência relativamente a direção do voo do satélite. Os gráficos (b), (c) e (d) representam a densidade de energia do espectro de ondas recuperado obtido de espectro de imagem SAR gerados de espectros de ondas de referência com direções principais de $0^{\circ}, 45^{\circ}$ e $90^{\circ}$.

\subsection{Período de Pico}

Na Figura 4, cada curva corresponde ao desvio do período de pico do espectro de ondas recuperado relativamente ao espectro de ondas de referência - definido por (12) - em função da direção principal do espectro de ondas inicial. As curvas estão inteiramente entrelaçadas, não configurando nenhum comportamento bem definido. Nota-se, porém, que o desvio do período de pico não é, de um modo geral, maior que $8 \%$, seja qual for a direção principal do espectro de ondas de referência considerado na simulação. Isto quer dizer que os períodos de pico dos espectros recuperados afastam-se, em média, de 1 segundo do valor do período de pico do espectro de ondas de referência. Conclui-se, portanto, que o período não é influenciado pela variação de direção principal do espectro de ondas inicial.

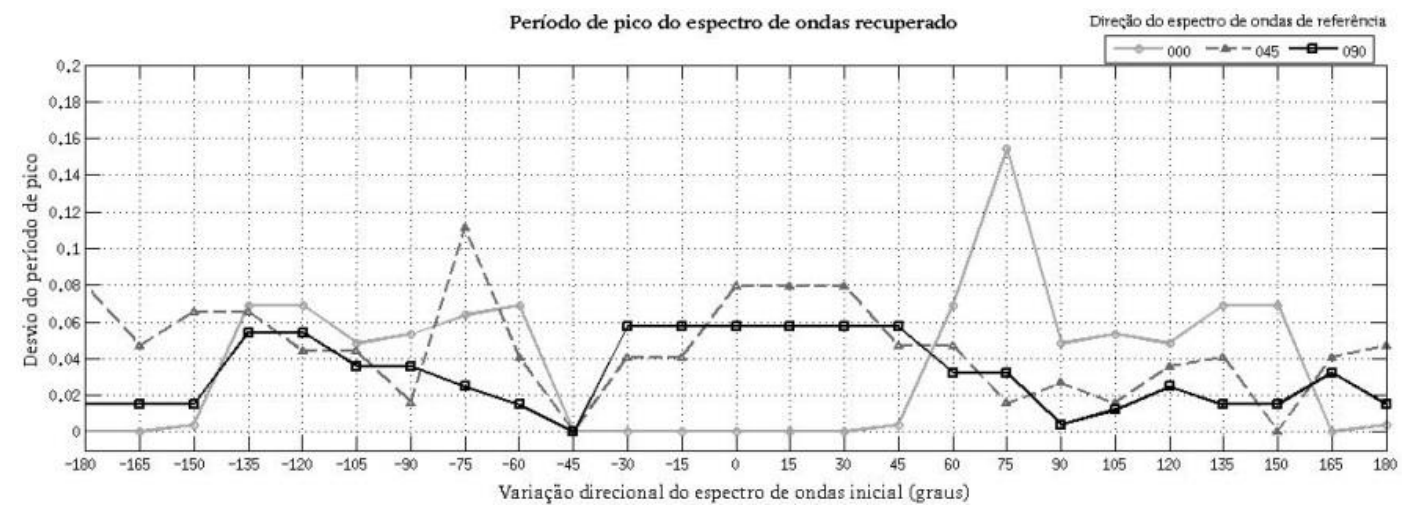

Figura 4: Representação da variação do desvio do período de pico (adimensional) do espectro de ondas recuperado em função da direção principal do espectro de ondas inicial e da direção principal do espectro de referência relativamente a direção do voo do satélite.

\subsection{Correlação Espectral}

Na Figura 5, cada curva corresponde ao coeficiente de correlação entre o espectro de ondas de referência e o espectro de ondas recuperado, definido por (7), em função da direção principal do espectro de ondas inicial. Todas as curvas possuem um aspecto comum: os maiores valores do coeficiente de correlação $(\geq 0.80)$ correspondem às menores variações angulares $\left(\left|\Delta^{0}\right| \leq 35^{\circ}\right)$. Isto quer dizer que o espectro de ondas de referência e o espectro de ondas recuperado possuem alto grau de similaridade. À medida que $\left|\Delta^{0}\right|$ aumenta, fica evidente que o grau de similaridade decresce drasticamente, acarretando que os espectros de ondas recuperados sejam muito diferentes do espectro de ondas de referência. Neste sentido, esta análise é conclusiva: o espectro de ondas recuperado depende fortemente da variação angular $\Delta^{0}$. 


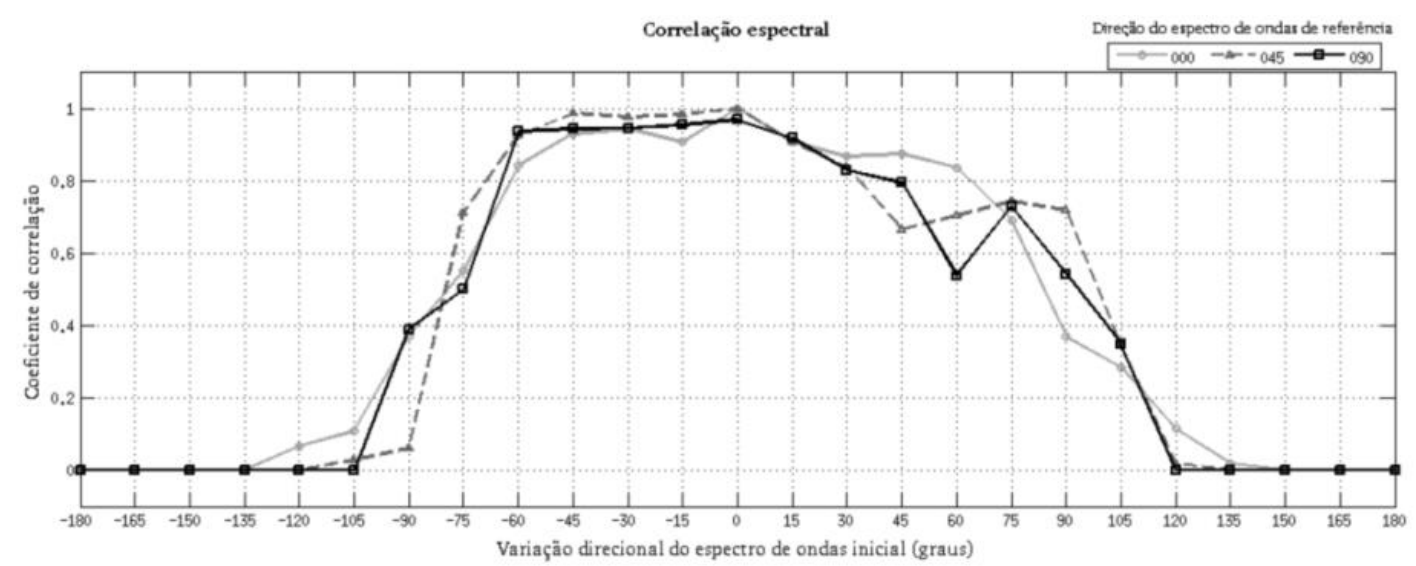

Figura 5: Representação da variação do coeficiente de correlação (adimensional) entre o espectro de ondas recuperado e o espectro de ondas de referência em função da direção principal do espectro de ondas inicial e da direção principal do espectro de referência relativamente a direção do voo do satélite.

$\mathrm{Na}$ análise feita a seguir, tenta-se estabelecer uma relação (percepção visual) do espectro de ondas recuperado e o seu grau de correlação com o espectro de ondas de referência. Partindose do espectro de ondas de referência com direção principal $45^{\circ}$, visto na Figura 6 , gera-se o espectro de imagem SAR apresentado na Figura 7, levando em conta a adição de um ruído aleatoriamente produzido. Este espectro de imagem SAR, correspondente à cena descrita pelo espectro de ondas de referência da Figura 6, será utilizado para recuperação de espectros. Os resultados obtidos pelo algoritmo MPI serão analisados quando as seguintes rotações do espectro de ondas de referência forem usadas como espectro de ondas inicial: $\Delta^{0}=$ : (i) $-30^{\circ}$; (ii) $0^{\circ}$; (iii) $45^{\circ}$; (iv) $-90^{\circ}$; (v) $-105^{\circ}$ e (vi) $150^{\circ}$.

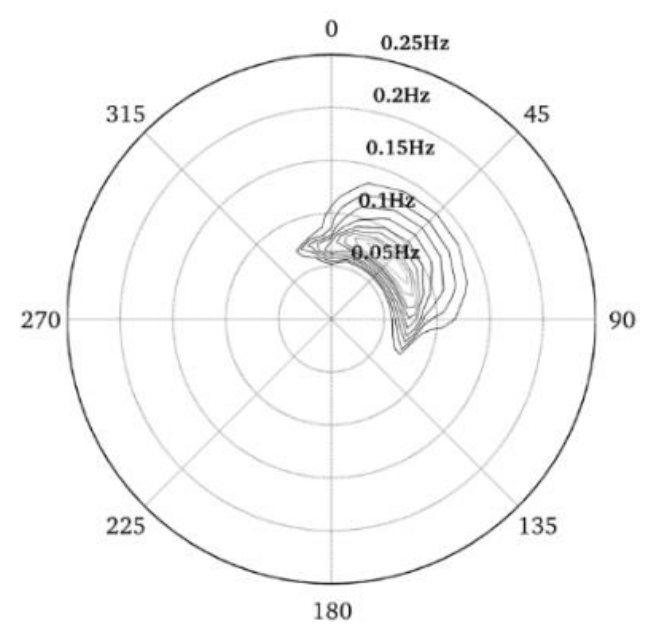

Figura 6: Espectro direcional de ondas (JONSWAP $x \cos ^{2 s}$ ) com direção principal de $45^{0}$, utilizado como espectro de ondas de referência numa das simulações. 


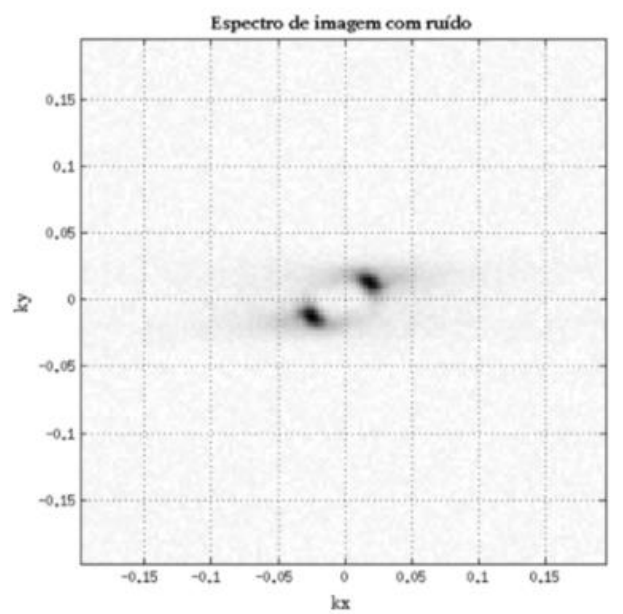

Figura 7: Espectro de imagem SAR com direções $45^{0}$ e $225^{0}$ (ambiguidade) gerado a partir do espectro de ondas referência da Figura 6.

(i) Neste caso o espectro de ondas inicial possui direção principal de $15^{\circ}$. O coeficiente de correlação entre o espectro de ondas recuperado e o espectro de ondas de referência é alto, aproximadamente 0.98. Isto garante grande similaridade entre estes espectros direcionais. Este fato é constatado rapidamente com uma inspeção visual entre os itens (a) e (c) da Figura 8. É importante observar que a direção principal do espectro de ondas recuperado é preservada, isto é, persiste muito proximamente a $45^{\circ}$, enquanto que a direção média sofre influência grande da direção principal do espectro de ondas inicial. Isto é percebido pela leve rotação (que é uma distorção) imprimida ao espectro de ondas recuperado se comparado ao espectro de ondas de referência.

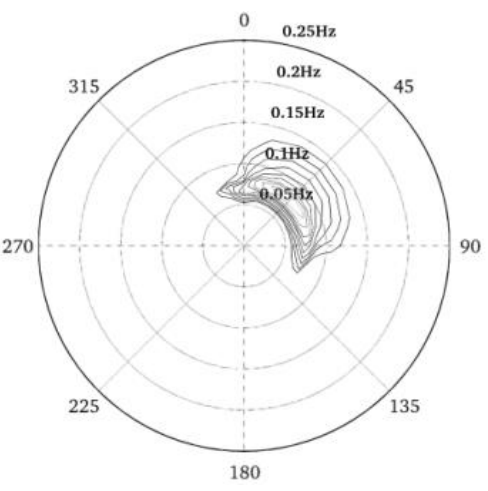

(a)

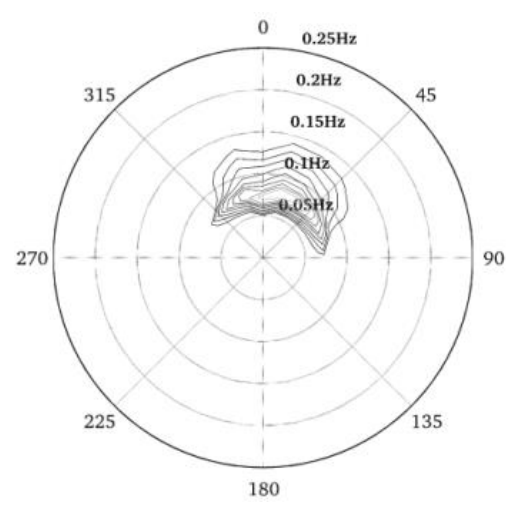

(b)

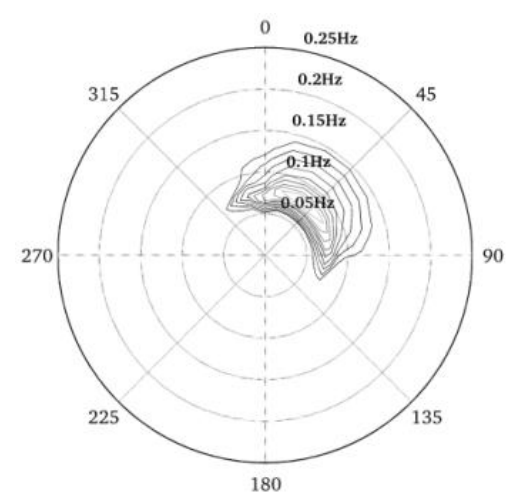

(c)

Figura 8: Caso $\Delta^{0}=-30^{\circ}$ : (a) espectro de ondas de referência; (b) espectro de ondas inicial; (c) espectro de ondas recuperado.

(ii) Neste caso, o espectro de ondas inicial é o próprio espectro de ondas de referência. $\mathrm{O}$ coeficiente de correlação entre o espectro de ondas recuperado e o espectro de ondas de referência é igual a 1. Isto garante que o espectro de ondas recuperado e o espectro de ondas de referência estão totalmente correlacionados. A comparação entre os itens (a) e (c) da Figura 9 não deixa dúvida quanto a esta conclusão. 


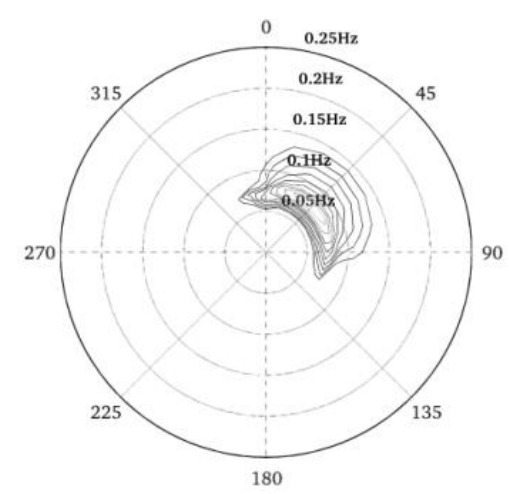

(a)

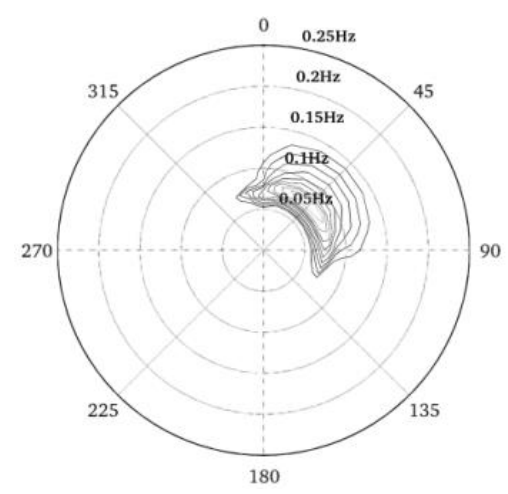

(b)

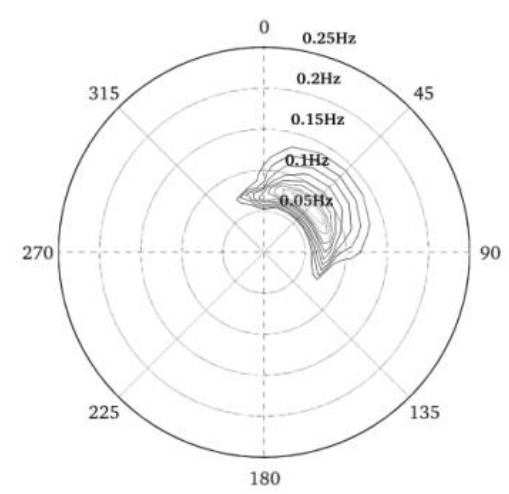

(c)

Figura 9: Caso $\Delta^{0}=0^{\circ}$ : (a) espectro de ondas de referência; (b) espectro de ondas inicial; (c) espectro de ondas recuperado.

(iii) Neste caso, o espectro de ondas inicial possui direção principal de $90^{\circ}$. O coeficiente de correlação entre o espectro de ondas recuperado e o espectro de ondas de referência é igual a 0.68. Uma inspeção visual constata que, de fato, a similaridade entre o espectro de ondas de referência e o espectro de ondas recuperado é bem menor. Como já observado, o espectro de ondas inicial impacta significativamente a direção média. Este fato pode ser reconfirmado comparando-se o espectro de ondas recuperado (Figura 10-c) com o espectro de ondas de referência (Figura 10-a): a direção média do espectro de ondas recuperado tende a acompanhar a direção principal do espectro de ondas inicial $\left(90^{\circ}\right)$.

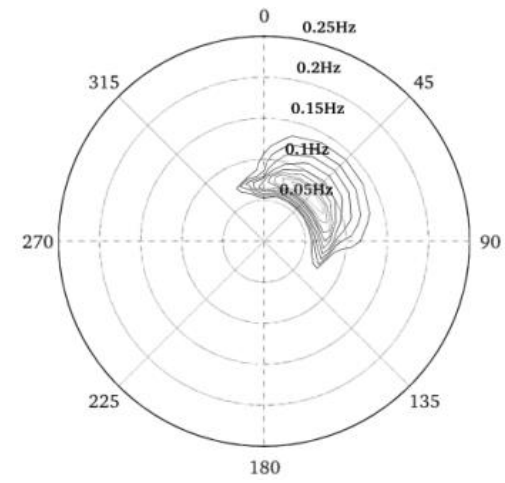

(a)

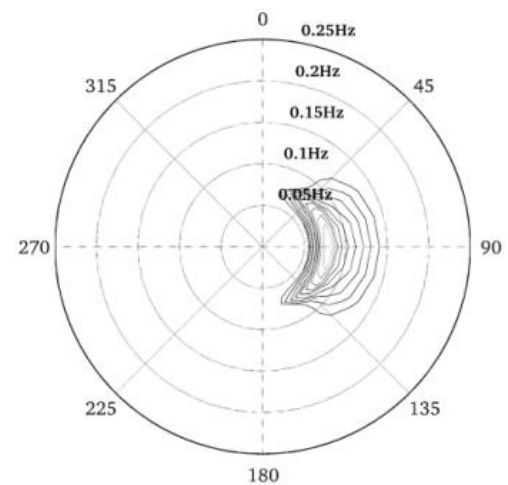

(b)

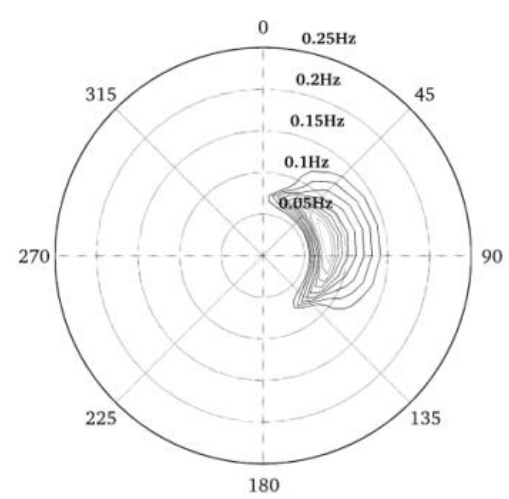

(c)

Figura 10: Caso $\Delta^{0}=45^{\circ}$ : (a) espectro de ondas de referência; (b) espectro de ondas inicial; (c) espectro de ondas recuperado.

(iv) Neste caso, o espectro de ondas inicial possui direção principal de $315^{\circ}$. O coeficiente de correlação entre o espectro de ondas recuperado e o espectro de ondas de referência decresce bastante e vale algo em torno de 0.06. A comparação visual entre os itens (a) e (c) da Figura 11 explica esta baixa correlação: a distorção é muito grande. Isto mostra em que medida uma escolha muito equivocada da direção principal do espectro de ondas inicial impacta os resultados obtidos. No presente caso e em outros não apresentados aqui, em que o espectro de ondas inicial possui direção principal aproximadamente ortogonal à direção do espectro de ondas de referência, a resposta exibida pelo mecanismo de inversão foi, invariavelmente, um 
espectro direcional particionado, o que corresponde a um sistema bimodal de ondas. Isto ocorre devido à baixa confiabilidade do espectro de ondas inicial em relação ao espectro de imagem, não sendo o mecanismo, portanto, capaz de decidir sobre a ambiguidade direcional com clareza. Por fim, cabe destacar que apesar da grande distorção entre os espectros em questão, o coeficiente de correlação entre eles só não é nulo devido ao sistema de ondas com direção principal próxima a $0^{\circ}$, que ainda preserva alguma característica do espectro de ondas de referência.

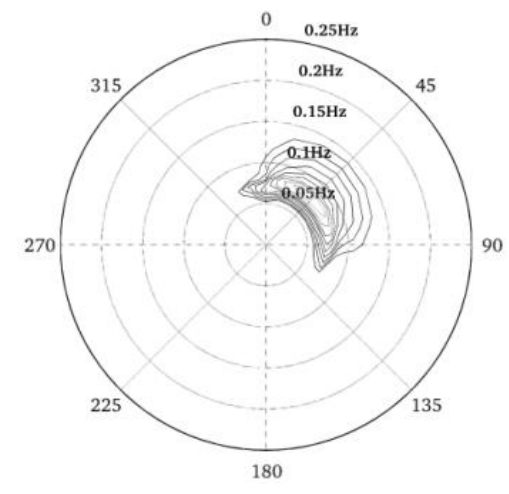

(a)

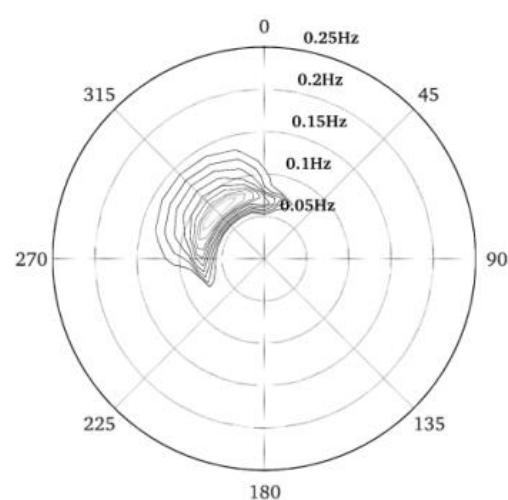

(b)

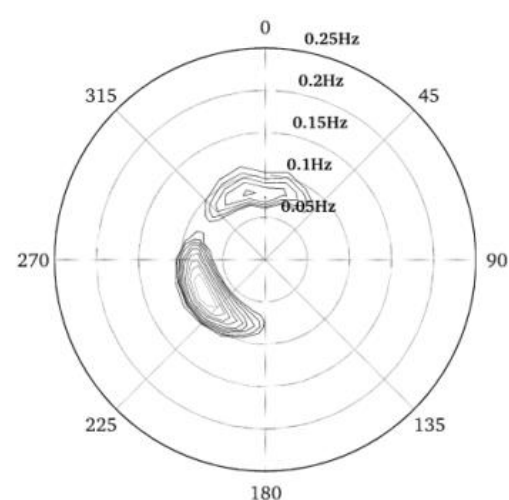

(c)

Figura 11: Caso $\Delta^{0}=-90^{\circ}$ : (a) espectro de ondas de referência; (b) espectro de ondas inicial; (c) espectro de ondas recuperado.

(v) Neste caso, o espectro de ondas inicial possui direção principal de $300^{\circ}$. O coeficiente de correlação entre o espectro de ondas recuperado e o espectro de ondas de referência é ainda menor: vale algo em torno de 0.028. A comparação visual entre os itens (a) e (c) da Figura 12 dá uma boa ideia sobre o baixo grau de similaridade entre os espectros direcionais em questão. Note-se que, como no caso (iv), há uma partição do espectro de ondas recuperado, pois a direção do espectro de ondas inicial é aproximadamente ortogonal à direção do espectro de imagem. $\mathrm{O}$ espectro de ondas recuperado neste caso guarda ainda menos características do espectro de ondas de referência, por isso seu grau de similaridade com o espectro de ondas de referência é ainda menor que o observado no caso (iv). É ilustrativo comparar os espectros de ondas recuperados nos casos (iv) e (v) tendo em mente a energia associada a cada um dos sistemas de ondas com direção principal próxima a $0^{\circ}$. Eles permitem compreender o decréscimo do coeficiente de correlação que ocorre nestes casos. 


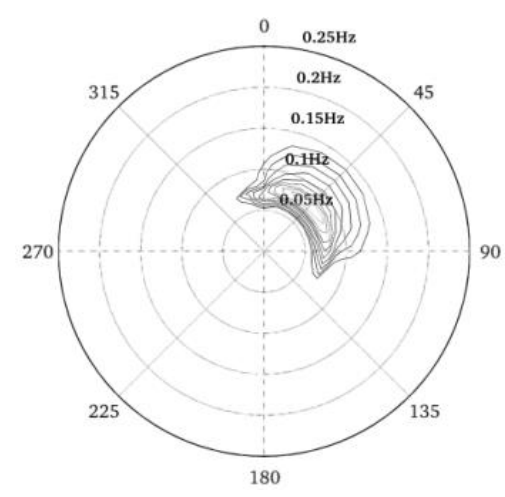

(a)

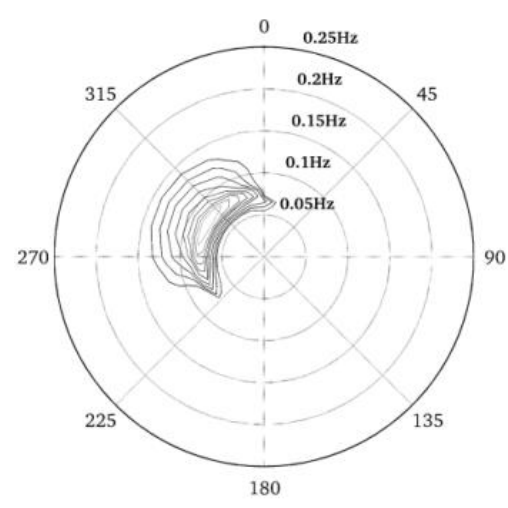

(b)

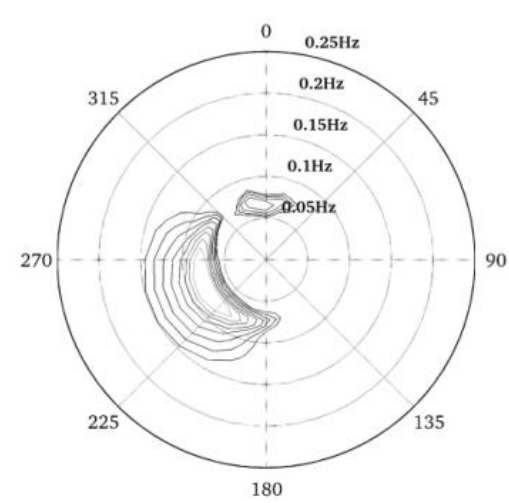

(c)

Figura 12: Caso $\Delta^{0}=-105^{\circ}$ : (a) espectro de ondas de referência; (b) espectro de ondas inicial; (c) espectro de ondas recuperado.

(vi) Neste caso, o espectro de ondas inicial possui direção principal de $195^{\circ}$. O coeficiente de correlação entre o espectro de ondas recuperado e o espectro de ondas de referência é zero, ou seja, não há correlação entre os espectros direcionais. A comparação visual entre os itens (a) e (c) da Figura 13 deixa muito claro que o espectro de ondas recuperado não guarda característica alguma do espectro de ondas de referência. Este resultado decorre da escolha de um espectro de ondas inicial com direção principal praticamente oposta à direção principal da direção do espectro de ondas de referência, levando o algoritmo de inversão basear-se nesta informação equivocada. $\mathrm{O}$ espectro de ondas de referência é o espelhamento deste espectro de ondas recuperado no primeiro quadrante.

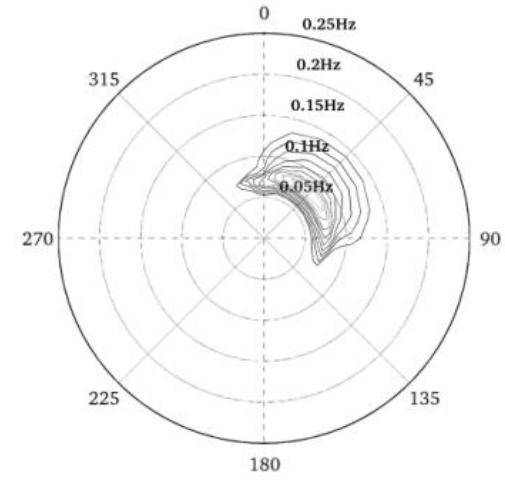

(a)

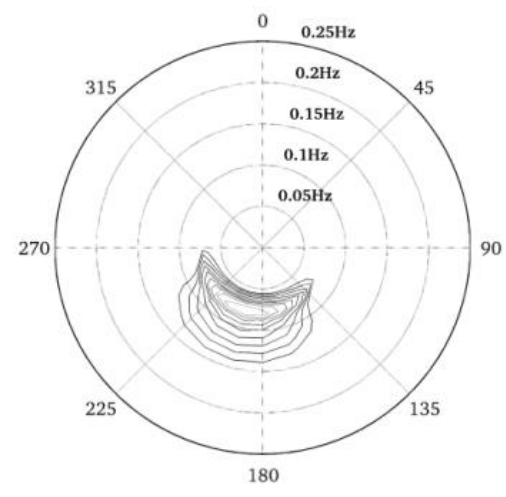

(b)

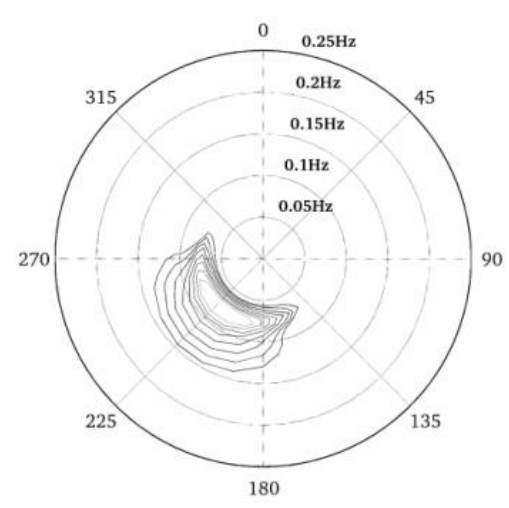

(c)

Figura 13: Caso $\Delta^{0}=150^{\circ}$ : (a) espectro de ondas de referência; (b) espectro de ondas inicial; (c) espectro de ondas recuperado. 


\section{CONCLUSÕES}

Neste artigo foi proposta uma nova metodologia para avaliar os impactos sofridos pelo espectro de ondas recuperado pelo SAR decorrentes da mudança da direção principal do espectro de ondas inicial. Os resultados obtidos para variações angulares da direção principal do espectro de ondas inicial em relação à direção principal do espectro de ondas de referência de até $60^{\circ}$ têm boa concordância com os reais valores dos parâmetros de onda utilizados como referência, além de alto grau de similaridade com o espectro de ondas de referência.

Para o estado de mar considerado, constata-se que a direção média das ondas do espectro de ondas recuperado é muito influenciada pela direção principal do espectro de ondas inicial. Diferentemente, a direção principal das ondas do espectro de ondas recuperado mostra-se bastante estável, desde que a direção do espectro de ondas inicial seja escolhida em uma das vizinhanças da direção do espectro de imagem SAR. Observa-se grande instabilidade deste parâmetro se a direção principal do espectro de ondas inicial é aproximadamente ortogonal à direção do espectro de imagem SAR. Isto não chega, entretanto, a ser uma surpresa, uma vez que um espectro de ondas inicial nestas condições não resolve a ambiguidade direcional.

O período de pico (assim como o período médio) não apresenta um comportamento bem definido nas simulações realizadas. Entretanto, este desvio mostrou-se limitado a, no máximo, $8 \%$. Isto corresponde a, aproximadamente, 1 segundo de defasagem em relação período do espectro de ondas de referência. Os resultados sobre os desvios da altura significativa não revelam, a princípio, um comportamento muito bem definido. Indicam, porém, que o desvio

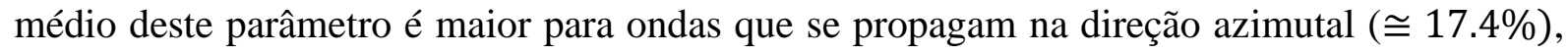

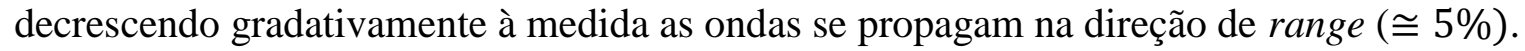

$\mathrm{O}$ mais importante resultado deste trabalho diz respeito à energia espectral. A energia do espectro de ondas recuperado correspondente a determinado estado de mar varia em função da direção principal do espectro de ondas inicial e da direção de propagação das ondas do sistema de ondas considerado. Especificamente, constatou-se que os maiores valores da densidade de energia do espectro de ondas recuperado são detectados quando a direção principal do espectro de ondas inicial tende a alinhar-se à direção do espectro de imagem SAR observado. De fato, os maiores valores da densidade de energia são verificados para sistemas de ondas que se deslocam paralelamente à direção de voo do satélite (azimutal), enquanto que para ondas que se propagam nas outras direções consideradas, estes valores são menos intensos. Isto indica como propor modificações acerca da energia do espectro de ondas inicial de forma a recuperar espectros de ondas com parâmetros de onda mais acurados e com maior correlação com o espectro de ondas de referência. Esta discussão sobre a energia espectral revela que a altura significativa é bastante sensível à variação da direção principal do espectro de ondas inicial, assim como é bem determinado o seu comportamento.

Conclui-se, portanto, que esta metodologia mostra-se muito promissora, pois com as devidas adaptações, ela pode ser utilizada na análise de sensibilidade do espectro de ondas recuperado a outros parâmetros de onda. Sua aplicabilidade pode ser facilmente estendida a situações reais, bastando para isto uma devida adequação. Sendo assim, é possível estimar erros na obtenção de parâmetros de ondas através do SAR e detectar como e quais fatores envolvidos neste processo afetam o resultado obtido. 


\section{AGRADECIMENTOS:}

Agradecimento a CAPES pela concessão de bolsa de estudo para Felipe Marques dos Santos, aluno do curso de doutorado do PPG-EM/UERJ.

\section{REFERÊNCIAS BIBLIOGRÁFICAS}

Abdalla S., Bidlot, J. R., Janssen, P. Global validation and assimilation of Envisat ASAR wave mode spectra. Proc. of SEASAR, Frascati, Italy, p. 1-8, 2006.

Aouf, L., Lefèvre, J-M., Hauser, D. Assimilation of Directional Wave Spectra in the Wave Model WAM: An Impact Study from Synthetic Observations in Preparation for the SWIMSAT Satellite Mission. J. Atm. and Oceanic Technology, 23, p. 448-463, 2006.

Cartwright, M. Fourier Methods for Mathematicians, Scientists and Engineers. United Kingdon, Ellis Horwood, p. 179-180, 1990

Collard, F., F. Ardhuin, B. Chapron. Extraction of coastal ocean wave fields from SAR images, IEEE Journal of Oceanic Engineering, 30(3): 526-533, 2005

Corsini, G. E Manara, G. Sea wave spectrum estimation from SAR images: a simulation based approach. Geoscience and Remote Sensing, 2: 936 - 938, 1995

Hasselmann, K. E Hasselmann, S. On the nonlinear mapping of an ocean wave spectrum into a synthetic aperture radar image spectrum and its inversion. Journal of Geophysical Reasearch, 96: 713 - 729. 1991.

Heiberg, H., Breivik, L-A., Reistad, M., Brattli, A. Use of ASAR wave spectra in operational wave analysis and forecasting, report 10, Norwegian Meteorological Institute. 2006.

Kasilingam, D.P. E Shemdin, O. H. Models for synthetic aperture radar imaging of the ocean: A comparison. Journal of Geophysical Research 95: 1010 -1029. 1990.

Kahma, K., Hauser, D., Krogstad, H., Lehner, S., Monbaliu, J., Wyatt, L. Measuring and analyzing the directional spectra of ocean waves, EU COST Action 174, EUR 21367, p. 19-20, 2005.

Komen, G. J., Cavaleri, L., Donelan, M., Hasselmann, K., Hasselmann S., Janssen, P. Dynamics and modelling of ocean waves. New York, Cambridge University Press, p. 390 - 393. 1994.

Krogstad, H. E Shyberg, H. On Hasselamann's nonlinear ocean-SAR transformation. Journal of Geophysical Reasearch, 97: 2421 - 2425, 1992.

Litovchenko, K. E Ivanov, A. Comparison of ERS-1 and ALMAZ-1 SAR ocean wave imaging. Geoscience and Remote Sensing, 3: 937 - 939. 1993.

Longuet-Higgins, M. S. The statistical analysis of a random, moving surface. Phil. Trans. R. Lond, 249: 321-387. 1957.

Phillips, O. M. The Dynamics of the Upper Ocean, Cambridge University Press, p. 99 - 103. 1977.

Robinson, I. S. Measuring the Oceans from Space. Great Britain, Springer-Praxis Books, p. 669. 2004. 
Stewart, D. E. Dynamics with Inequalities. Philadelphia, SIAM, p. 41. 2011.

Violante-Carvalho, N., Robinson, I., Gommenginger, C., Carvalho, L.M., F. Ocampo-Torres, J. The effect of the spatially inhomogeneous wind field on the wave spectra employing an ERS2 SAR PRI image, Continental Shelf Research (CRS), 36: 1-7. 2012.

WAFO-group (2000). "WAFO - A Matlab Toolbox for Analysis of Random Waves and Loads - A Tutorial" Math. Stat., Center for Math. Sci., Lund Univ., Lund, Sweden. Accessed 08/12/2014. http://www.maths.lth.se/matstat/wafo.

Wang, H ; Zhu, Jh ; Yang, Js ; Shi, Cy. A semiempirical algorithm for SAR wave height retrieval and its validation using Envisat ASAR wave mode data, Acta Oceanologica Sinica, 2012, 31(3): 59-66. 2012.

(Recebido em setembro de 2014. Aceito em dezembro de 2014). 